\title{
Prospective Study of the Frequency and Outcomes of Patients with Suspected Pulmonary Embolism Administered Heparin Prior to Confirmatory Imaging
}

\author{
Jeffrey A. Kline, MD \\ Carolinas Medical Center, Charlotte, NC. Jkline@carolinas.org \\ Michael R. Marchick, MD \\ University of Florida, Gainesville, FL marchick@ufl.edu \\ Christopher Kabrhel, MD \\ Massachusetts General Hospital, Boston, MA. ckabrhel@partners.org \\ D. Mark Courtney, MD \\ Northwestern University, Chicago, IL. mcourtney@nmff.org
}

\section{Summary}

Objectives-The administration of empiric systemic anticoagulation (ESA) before confirmatory radiographic testing in patients with suspected pulmonary embolism (PE) may improve outcomes, but no data have been published regarding current practice. We describe the use of ESA in a large prospective cohort of emergency department (ED) patients and report the outcomes of those treated with ESA compared with patients not receiving ESA.

\begin{abstract}
Methods-12-center, noninterventional study of ED patients who presented with symptoms concerning for PE. Clinical data including pretest probability and decision to start ESA were recorded at point of care by attending physicians. Patients were followed for adverse in-hospital outcomes and recurrence of venous thromboembolism.
\end{abstract}

\begin{abstract}
Results-ESA was initiated 342/7932 (4.3\%) of enrolled patients, including 142/618 (23\%) patients with high pretest probability. Patients receiving ESA had more abnormal vital signs and were more likely to have a history of venous thromboembolism than those who did not receive ESA. Overall, 481/7,932 (6.1\%) had PE diagnosed, 72/481 (15.0\%) with PE had ESA, and 72/342 $(21 \%)$ of ESA patients had PE. Three patients $(0.9 \%, 95 \%$ CI: $0.2-2.5 \%)$ who received ESA suffered hemorrhagic complications compared with 38 patients $(0.5 \%, 95 \% \mathrm{CI}: 0.4-0.7 \%)$ who did not receive ESA.
\end{abstract}

\footnotetext{
(C) 2011 Elsevier Ltd. All rights reserved

Correspondence: Jeffrey A. Kline, MD Emergency Medicine Research Department of Emergency Medicine Carolinas Medical Center PO Box 32861 Charlotte, NC 28323-2861 jkline@carolinas.org Phone 704 355-7092 Fax 704 355-7047.

Publisher's Disclaimer: This is a PDF file of an unedited manuscript that has been accepted for publication. As a service to our customers we are providing this early version of the manuscript. The manuscript will undergo copyediting, typesetting, and review of the resulting proof before it is published in its final citable form. Please note that during the production process errors may be discovered which could affect the content, and all legal disclaimers that apply to the journal pertain.

Author contributions JAK organized the study, obtained funding, collected and analyzed data and drafted and revised the manuscript. MRM conceived the present analysis of this cohort, analyzed data and drafted the manuscript DMC, CK, participated in writing the study protocol, obtaining funding, data collection and revising the manuscript.

Conflicts of interest and Financial disclosure JAK owns stock in CP Diagnostics LLC and Studymaker LLC

All authors have access to all data using the rules of the data use agreement
} 
Conclusions-In this multicenter sample, ED physicians administered ESA to a small, generally more acutely ill subset of patients with high pretest probability of PE, and very few had hemorrhagic complications. ESA was not associated with any clear difference in outcomes. More study is needed to clarify the risk versus benefit of ESA.

\section{Keywords}

Pulmonary embolism; empiric anticoagulation; decision-making; venous thromboembolism

\section{Introduction}

Delay in the initiation of anticoagulation for patients with diagnosed pulmonary embolism (PE) has been associated with higher rates of venous thromboembolism (VTE) recurrence and worsened patient outcomes including disability and death. ${ }^{1-6}$ In an urban teaching hospital with 24 hours per day, 7 days per week imaging capability, Kline et al found the median time duration between patient presentation and administration of heparin was 8 hours for patients who had PE diagnosed in the ED versus 33 hours for patients with delayed diagnosis. ${ }^{5}$ Prior work has proposed that the benefit of empiric systemic anticoagulation (ESA, i.e., the administration of heparin prior to knowing the results of confirmatory diagnostic testing) outweighs the risk in patients who engender a high pretest probability for PE. ${ }^{7}$ However, to our knowledge, no prior investigation has examined the current practice patterns and associated patient outcomes for ESA in a cohort of patients with suspected PE.

This study examines a large cohort of patients presenting to 12 EDs with symptoms concerning for PE using methodology specifically designed to examine the frequency of ESA use and patient outcomes. We hypothesized that ESA would be reserved disproportionately for a subset of severely ill patients with high pretest probability of PE and that the use of ESA would be associated with a low incidence of hemorrhagic complications.

\section{Methods}

\section{Study Design and Setting}

This was a secondary analysis of a prospective cohort of patients presenting to 12 EDs who were evaluated for PE. ${ }^{8}$ Study sites included 10 academic centers and 2 community hospitals in the United States. Patients were enrolled from July 1, 2003 through November 30, 2006. The study protocol was approved by the Institutional Review Boards of each participating hospital.

\section{Study Population}

For a patient to be eligible for enrollment, a physician was required to have sufficient suspicion for PE to order objective diagnostic testing in the ED. This included D-dimer measurement, CT pulmonary angiography, ventilation/perfusion (VQ) scanning or venous ultrasonography. We specifically required that this testing be performed with the intent of evaluating for acute PE and excluded patients who underwent testing solely for suspected deep venous thrombosis (DVT) or other conditions.

Study centers had the option of enrolling patients during randomly selected 8 hour periods or consecutively with a goal of enrollment of $>85 \%$ of eligible subjects. To ensure compliance with the $85 \%$ goal, centers underwent quarterly audits of all D-dimer testing, VQ scans and CT pulmonary angiograms obtained during enrollment periods via retrospective review of ordering databases. 


\section{Measurements}

Data were entered at the point of care by ED physicians into a web-based, secure, electronic data collection form. This protocol specified that the form be completed before the results of confirmatory diagnostic testing for PE were known. A full description of the content and methodology for this data collection system has been published. ${ }^{9}$ Explicit definitions were provided for each data field. Automated checks were employed to prevent upload of missing or nonsensical data. Forms could be initiated at any time after a test was ordered for PE, and the form could be edited for up to 24 hours after upload. The primary predictor for this analysis was administration of ESA. The field used to determine ESA status was the seventh out of 74 questions, immediately after a set of questions used to estimate the Wells score and the physician's unstructured (gestalt) estimate of the pretest probability of PE. ${ }^{10 ; 11}$ The ESA field required the clinician to state whether or not anticoagulation (heparin or low molecular weight heparin) was given prior to the performance of diagnostic imaging for VTE.

Physicians were also asked to document the presence of absence of contraindications to anticoagulation with heparin. Absolute contraindications included any history of intracranial or intraocular hemorrhage; ischemic stroke within the previous 3 months; presence of a cerebral vascular malformation or intracranial metastasis; suspected aortic dissection; history of heparin induced thrombocytopenia; known bleeding diathesis; and uncontrolled hemorrhage. Relative contraindications included: uncontrolled hypertension (systolic blood pressure greater than $180 \mathrm{mmHg}$ and/or diastolic blood pressure greater than $110 \mathrm{mmHg}$ ); intracranial pathology not constituting an absolute contraindication; major surgery within the preceding 3 months; internal bleeding within the previous 4 weeks; active peptic ulcer disease; non-compressible vascular punctures; and allergy to heparin. Clinicians who completed these forms received standardized instruction, administered by a full-time study coordinator who traveled to each site to initiate the study. We performed quality assurance testing on a convenience audit of 1022 uploaded forms compared against the medical record and found $98 \%$ agreement between the answer coded in the ESA field and any anticoagulation orders on the medical record. ${ }^{9}$ The dose, route and type of anticoagulation were not specifically recorded.

The diagnosis of PE in this study required either a CT angiogram or conventional pulmonary angiogram demonstrating a pulmonary arterial filling defect interpreted as positive for acute PE, a high probability VQ scan, or autopsy positive for PE. Imaging studies were interpreted by board certified radiologists not affiliated with the study. Patients were followed in hospital for hemorrhagic complications, ICU admission and death, and to 45 days for recurrence of PE or DVT. The data collection form allowed the hemorrhage site to be determined as 1. Intracranial, 2. Gastrointestinal, 3. Puncture site, or 4. Other. .All sites underwent an initiation visit from the study monitor that included a verbal and written explanation of how follow-up variables were defined. A bleeding event was recorded as present if the event caused a clinically significant change in care, including stopping or reversal of anticoagulation, or a transfusion, or surgical or endoscopic intervention

\section{Data Analysis}

Patients were grouped by presence or absence of ESA and presence or absence of PE at the time of initial presentation. Two $\times 2$ contingency tables containing frequencies of adverse outcomes among each group were constructed.

Continuous data are described as mean \pm standard deviation (SD) or median (intraquartile range [IQR] as appropriate. Categorical data are presented as percentages along with the 95\% confidence interval (CI) when appropriate (Clopper-Pearson method). Multivariate logistic regression was performed using demographic information and a set of 15 additional characteristics (listed in Table 2) hypothesized to have an effect on clinicians' decision to 
administer ESA. Post-hoc power calculation was performed to determine the study's ability to detect both a difference in the rate of hemorrhagic complications and a difference in the rate of VTE recurrence. Using the entire cohort of patients (regardless of presence or absence of PE), and setting $\alpha=0.05$ and $\beta=0.20$, the study was able to detect a $1.5 \%$ difference in the rate of hemorrhagic complications in those administered ESA compared with those not receiving ESA. Among patients initially diagnosed with PE, the study was powered to detect a difference in VTE recurrence of 9\% between those receiving ESA and those who did not. Statistical analyses were performed using StatsDirect v. 2.6.2 (StatsDirect, Cheshire, England).

\section{Results}

We enrolled 7,932 patients with necessary data to assess use of ESA and determine outcomes in accordance with the protocol. A total of 342 patients $(4.3 \%)$ received anticoagulation prior to any confirmatory diagnostic testing. Ninety-one $(26 \%)$ of these patients received low molecular weight heparin and the others received unfractionated heparin. We did not record dosing information. Table 2 compares the characteristics of all patients based upon use or non-use of ESA. Multivariate regression revealed that patients given ESA were older, more likely to be male, have a personal or family history of VTE, and have a higher heart rate, respiratory rate, and Wells score, and a lower oxygen saturation and systolic blood pressure. Curiously, the frequency of relative or absolute contraindications to anticoagulation were not significantly different between patients who received ESA ( 22 of 342 or $6.4 \%$ ) versus patients not receiving ESA ( 418 of 7590 or $5.5 \%$, 95\% CI for difference of $0.9 \%$ : $-1.2 \%$ to $4.1 \%$ ).

From the initial cohort of 7,932 patients, 481 (6.1\%) were diagnosed with PE at the time of initial presentation. 72 (21.1\%) of the 342 patients receiving ESA were diagnosed with PE. Therefore, $15.0 \%$ of the patients diagnosed with $\mathrm{PE}$ received anticoagulation prior to imaging, and $85.0 \%$ patients with PE were not administered ESA. Patients receiving pretest anticoagulation were significantly more likely to be diagnosed with PE (OR: 4.68, 95\%CI: 3.49-6.22). 11 patients who received ESA and were not diagnosed with PE were eventually diagnosed with DVT.

Table 1 displays the proportion of patients belonging to each of the four possible therapeutic and diagnostic outcome groups (ESA+/PE+, ESA-/PE+, ESA+/PE-, ESA-/PE-) among those grouped as high (Wells score $>6$, or physician gestalt-estimated pretest probability $>40 \%$ ), medium (Wells Score 2-6, or estimated pretest probability 15-40\%) or low pretest probability (Wells score $<2$, or estimated pretest probability $<15 \%$ ). $22.2 \%$ of patients with Wells score $>6$ received ESA, compared with $23.0 \%$ of patients with high gestalt estimate probability $(95 \%$ CI for difference: $-5.7 \%-6.9 \%)$. Among the 618 patients that had a high pretest probability based on Wells score or physician gestalt, $74(12 \%)$ had an absolute or relative contraindication to anticoagulation and $425(69 \%)$ were admitted to the hospital.

Subsequently, we compared the outcomes of patients receiving ESA to those not receiving early anticoagulation. Table 3 shows the frequency of significant patient outcomes grouped by the use of ESA and presence or absence of PE. Regardless of the presence of absence of PE, patients receiving ESA were significantly more likely to be admitted to an intensive care unit $(12.6 \%$ vs. $2.8 \%, 95 \% \mathrm{CI}$ for difference: $6.7 \%-13.7 \%)$. The in-hospital mortality of ESA patients was also higher in the cohort as a whole $(3.2 \%$ vs. $1.0 \%, 95 \% \mathrm{CI}$ for difference: $0.8 \%-4.7 \%)$. VTE recurrence within 45 days was noted in 5/72 (6.9\%) of PE+ patients who received ESA and 16/409 (3.9\%) of PE+ patients who did not receive ESA. 
The incidence of in-hospital hemorrhagic complications was low. Among the cohort as a whole, $3 / 342$ patients $(0.9 \%)$ receiving ESA suffered a hemorrhagic complication compared with $38 / 7590(0.5 \%)$ who did not receive ESA (95\% CI for difference: $-0.2 \%-2.1 \%$ ). One of the three given ESA was diagnosed with PE and had puncture site bleeding. Of the two patients who received ESA, but were not subsequently diagnosed with PE, one of these patients was diagnosed with DVT and had gastrointestinal bleeding, while the other patient had no PE or DVT but had an a non-fatal bleeding complication specified as "other bleeding" which did not require admission to an intensive care unit. None of the three patients who had ESA and bleeding had a transfusion or required endoscopy or surgery for hemorrhage control.

Of the 38 without ESA, two had intracranial hemorrhage, 16 had gastrointestinal hemorrhage, eight had puncture site bleeding and 12 had bleeding at other sites. Two of the 38 received systemic fibrinolytic treatment and two others intrapulmonary fibrinolytic prior to their bleeding episode. Eleven of these 38 received a blood transfusion and five had endoscopy, but none underwent surgery to control hemorrhage.

\section{Discussion}

The authors provide this set of data to provide additional information about the patterns of use and patient outcomes associated empiric systemic anticoagulation for suspected pulmonary embolism. We believe this topic is important, because the clinical question of the risk and benefit of ESA often arises in the emergency department and in medical malpractice lawsuits. The previous literature examining the use of ESA for suspected VTE prior to imaging is quite limited. Prior outcome studies have been restricted to patients with confirmed VTE. ${ }^{1-6}$ Moreover, no previous studies has specifically examined the outcomes of patients based upon administration of systemic anticoagulation prior to confirmatory imaging. To our knowledge, this is the first study to examine the characteristics of a cohort patients with suspected PE who received ESA and to compare the outcomes of patients with and without ESA stratified by ultimate diagnosis of PE. This comparison is important because in our sample, $79 \%$ (72/342) of patients who receive ESA ultimately were found to not have PE. Thus, help determine whether ESA is harmful or helpful, it is important to measure outcomes of patients with suspected PE and who received ESA, but ultimately had no PE after diagnostic testing.

Our results indicate that emergency physicians administer ESA to fewer than $25 \%$ of patients with a high pretest probability for PE, regardless if obtained by Wells score or clinician gestalt. Patients receiving ESA engendered the perception of higher acuity, as evidenced by the higher rate of ICU admission. Patients receiving ESA who were not diagnosed with PE also had higher mortality rates. We interpret the vital sign and comorbidity data in Table 2 as evidence that this increase in mortality was secondary to the increased severity of the clinical presentation and underlying illness of patients who received ESA. Another important observation was the very low overall rate of significant hemorrhagic complications associated with ESA; indeed, less than $1 \%$ of patients (2/270) who received ESA--but ultimately had no PE--also suffered a bleeding complication.

Strengths of the present study include the large sample size and the number of centers, both academic and community, which participated in data collection. Treatment decisions for patients in this study were made at the discretion of the individual physicians, who could rely on their own gestalt, available clinical decision rules or any combination of these.

However, a lack of data regarding the dose, route and type of heparin used is a limitation of this study. Furthermore, there are no data pertaining to the time elapsed between initiation of 
treatment and the completion of confirmatory testing. Finally, as patients were not randomized, one cannot conclude a causal effect of ESA on patient outcomes.

Our data show no clear evidence of benefit or harm from the use of ESA in current practice. We believe these data allow two preliminary inferences: 1 . That ED physicians currently reserve ESA for very sick patients, which precludes the use of a noninterventional ("wild type") study to answer the question of whether ESA is harmful or helpful, and 2. The rate of hemorrhagic complications was low in patients administered ESA despite their high acuity of illness. We conclude that a randomized trial to test the impact of ESA on mortality in patients with moderate to high pretest probability for PE is warranted.

\section{Acknowledgments}

Supported by Grants from the National Institutes for Health, R41HL074415 and R42HL074415, K23HL077404 and R01 HL074384, and a Medical Student Award from the Emergency Medicine Foundation

\section{References}

(1). Smith SB, Geske JB, Maguire JM, et al. Early Anticoagulation is Associated with Reduced Mortality for Acute Pulmonary Embolism. Chest. 2010; 137(6):1382-1389. [PubMed: 20081101]

(2). Ota M, Nakamura M, Yamada N, et al. Prognostic significance of early diagnosis in acute pulmonary thromboembolism with circulatory failure. Heart \& Vessels. 2002; 17(1):7-11. [PubMed: 12434196]

(3). Hull RD, Raskob GE, Brant RF, et al. Relation between the time to achieve the lower limit of the APTT therapeutic range and recurrent venous thromboembolism during heparin treatment for deep vein thrombosis. Arch Intern Med. 1997; 157(22):2562-2568. [PubMed: 9531224]

(4). Hull RD, Raskob GE, Brant RF, et al. The importance of initial heparin treatment on long-term clinical outcomes of antithrombotic therapy. The emerging theme of delayed recurrence. Arch Intern Med. 1997; 157(20):2317-2321. [PubMed: 9361572]

(5). Kline JA, Hernandez J, Jones AE, et al. Prospective study of the clinical features and outcomes of emergency department patients with delayed diagnosis of pulmonary embolism. Acad Emerg Med. 2007; 14(7):592-598. [PubMed: 17554011]

(6). Jelinek GA, Ingarfield SL, Mountain D, et al. Emergency Department diagnosis of pulmonary embolism is associated with significantly reduced mortality: a linked data population study. Emerg Med Australas. 2009; 21(4):269-276. [PubMed: 19682011]

(7). Hogg KE, Brown MD, Kline JA. Estimating the pretest probability to justify the empiric admininistration of heparin prior to pulmonary vascular imaging for pulmonary embolism. Thromb Res. 2006; 118:547-553. [PubMed: 16356538]

(8). Courtney DM, Kline JA, KABRHEL C, et al. Clinical features from the history and physical examination that predict the presence or absence of pulmonary embolism in symptomatic emergency department patients: Results of a prospective, multicenter study. Ann Emerg Med. 2010; 55(4):305-315.

(9). Kline JA, Johnson CL, Webb WB, et al. Prospective study of clinician-entered research data in the emergency department using an internet-based system after the HIPAA Privacy Rule. BMC Medical Informatics and Decision Making 2004. 2005; 4(1):17-25.

(10). Wells PS, Anderson DR, Rodger M, et al. Derivation of a simple clinical model to categorize patients probability of pulmonary embolism: Increasing the models utility with the SimpliRED D-dimer. Thromb Haemost. 2000; 83(3):416-420. [PubMed: 10744147]

(11). Runyon MS, Webb WB, Jones AE, et al. Comparison of the unstructured clinician estimate of low clinical probability for pulmonary embolism to the Canadian score or the Charlotte rule. Acad Emerg Med. 2005; 12(7):587-593. [PubMed: 15995088] 


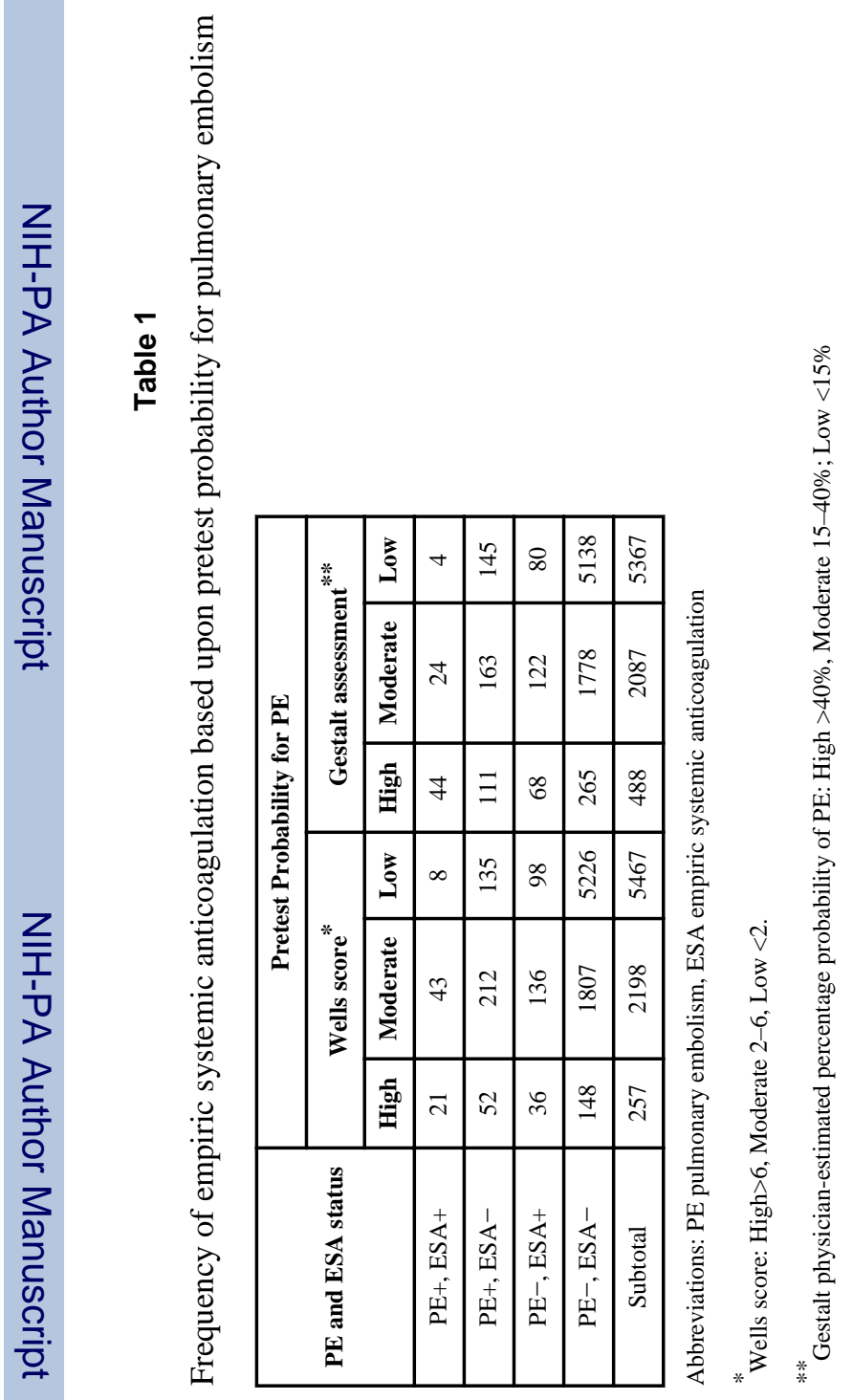

Thromb Res. Author manuscript; available in PMC 2013 April 1. 
Table 2

Patient characteristics by pretest treatment group

\begin{tabular}{|l|c|c|c|}
\hline Characteristic & ESA n=342 & No ESA n=7590 & P Value* \\
\hline Age (years) & $54.5 \pm 16.7$ & $48.7 \pm 17.3$ & 0.001 \\
\hline Female (\%) & $195(57)$ & $5130(68)$ & 0.001 \\
\hline White (\%) & $208(61)$ & $4327(57)$ & 0.14 \\
\hline Black (\%) & $107(31)$ & $2695(36)$ & 0.12 \\
\hline Hispanic (\%) & $24(7)$ & $458(6)$ & 0.08 \\
\hline Asian (\%) & $2(1)$ & $72(1)$ & 0.32 \\
\hline Highest HR (beats/min) & $101.2 \pm 24.8$ & $91.8 \pm 20.8$ & $<0.001$ \\
\hline Highest RR (breaths/min) & $23.3 \pm 5.8$ & $20.7 \pm 4.8$ & $<0.001$ \\
\hline Lowest SBP (mm Hg) & $126.0 \pm 25.7$ & $131.2 \pm 23.8$ & 0.02 \\
\hline Lowest SaO2 (\%) & $94.3 \pm 5.5$ & $96.6 \pm 3.7$ & $<0.001$ \\
\hline Wells Score & $3.5(1.5-6)$ & $1.5(0-3)$ & $<0.001$ \\
\hline Prior VTE (\%) & $87(25)$ & $770(10)$ & $<0.001$ \\
\hline Family History of VTE (\%) & $46(13)$ & $774(10)$ & 0.007 \\
\hline Malignancy $\dagger(\%)$ & $41(12)$ & $448(6)$ & 0.06 \\
\hline Immobility (\%) & $58(17)$ & $705(9)$ & 0.11 \\
\hline Exogenous Estrogen Use $(\%)$ & $27(8)$ & $634(8)$ & 0.10 \\
\hline Recent Surgery (\%) & $28(8)$ & $492(6)$ & 0.61 \\
\hline History of CHF $(\%)$ & $42(12)$ & $539(7)$ & 0.30 \\
\hline History of CAD (\%) & $48(14)$ & $785(10)$ & 0.85 \\
\hline History of COPD (\%) & $61(18)$ & $795(10)$ & 0.96 \\
\hline Any End-Stage Condition $(\%)$ & $13(4)$ & $146(2)$ & 0.76 \\
\hline
\end{tabular}

Data are displayed as mean $\pm \mathrm{SD}$ (or median (IQR) for Wells score) for continuous data and $\mathrm{n}(\%)$ for dichotomous characteristics. Abbreviations: HR: heart rate, RR: respiratory rate, SBP: systolic blood pressure, $\mathrm{SaO}$ 2, room air oxygen saturation, VTE: venous thromboembolism, CHF: congestive heart failure, CAD: coronary artery disease, COPD: chronic obstructive pulmonary disease. Immobility was defined as any condition which resulted in limited mobility of the patient, e.g. bed-bound or paralyzed patients, or a limb, e.g. use of a cast or external fixator, for a period of greater than 48 hours. End-stage condition was defined as any ailment resulting in a life expectancy of less than 6 months.

$\dagger$ Currently treated or metastatic malignancy

From multivariate logistic regression 


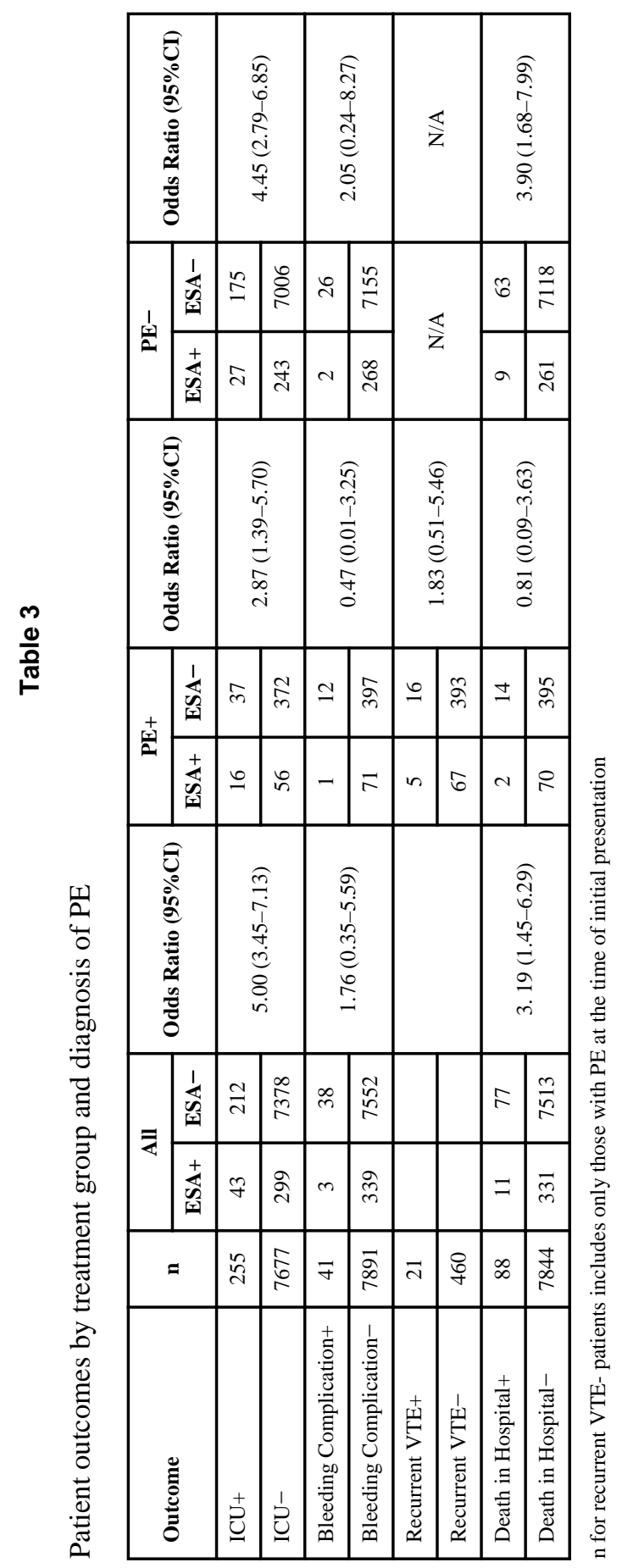

Thromb Res. Author manuscript; available in PMC 2013 April 1. 